\title{
EXCITATION OF DYNAMO MODES
}

\author{
P. HOYNG \\ Laboratory for Space Research \\ Beneluxlaan 21, 3527 HS Utrecht \\ The Netherlands
}

\begin{abstract}
After the very suggestive results of the early days, the theory of the solar dynamo has now entered a period of re-evaluation. It is clear that our initial expectations have been too high. I shall review some of the recent attempts to formulate nonlinear and stochastic mode excitation theoretically. We now have evidence from synoptic observations that the solar dynamo features many periods. Periods both shorter and longer than the fundamental 22 yr cycle have been claimed. The phase stability of any of these periods is uncertain. The phase memory of the 22 yr period may be as short as $\sim 10$ cycles, but could also be much longer. Linear mean field theories permit only one marginally stable mode; they predict one period with an infinitely long phase memory. Attempts to explain multiperiodicity and finite phase memory effects fall in two categories:

(1). Nonlinear models. These feature a few nonlinearly coupled variables and may exhibit a multiperiodic or chaotic behaviour; (2). If the number of relevant variables is very high, then the dynamo behaves stochastically. It has been argued that this takes the form of stochastic excitation of many dynamo modes (overtones).
\end{abstract}

\section{Introduction}

Dynamo theory of the solar cycle has made a very rapid progress in the period, say, 1965 - 1980. Many mean field dynamo models have been constructed during that time which reproduced a number of the basic features of the large-scale solar magnetic field. A number of problems had been recognised at an early stage, too (magnitude $\alpha$ coefficient, role of nonlinearities, $\mathrm{E}-\mathrm{W}$ orientation of bipolar sunspot groups, etc.), but there appeared to be a genuine optimism in those days that these would be solved in due course. In the meantime, (linear) mean field model building is out of fashion. And in retrospect it is clear that much of the successes of mean field theory, if very suggestive, were only apparent, and that our initial expectations had been pitched too high. As a result, dynamo theory of the solar magnetic field finds itself in a period of reappraisal. Two causes can be pinpointed for the crisis: (1). The required increase of the angular speed $\Omega$ with decreasing $r$ is neither found in selfconsistent simulations (Gilman and Miller 1981; Gilman 1983; Glatzmaier 1985a), nor measured by helioseismology (for a review see Harvey 1988).

(2). The validity of mean field theory is subject to a number of restrictions which have always been somewhat obscure. As a result, mean field modeling is often 
handled as an exercice in applied mathematics, with little regard for the physical restrictions and selfconsistency (the 'cookbook approach').

In recent years, attention has shifted from the convection zone to the boundary layer between convection zone and radiative interior as a more likely loeation for the solar dynamo (Spiegel and Weiss 1980; Galloway and Weiss 1981; Spruit and Van Ballegooijen 1982). A number of problems that beset a dynamo operating in the convection zone, among which in particular the wrong rotation curve mentioned under (1), may be defused in this way, see e.g. Glatzmaier (1985b). The arguments have been reviewed by Schüssler (1984), Stix (1987) and by Gilman et al. (1989). Dziembowski et al. (1989) report an inward decrease in $\Omega$ of about $20 \mathrm{nHz}$ in a $50000 \mathrm{~km}$ layer at $r / R=0.73$. If confirmed, this would provide a strong support for the idea of a boundary layer dynamo.

It is not my intention to discuss in detail the relative merits of specific dynamo models (see e.g. Parker 1987). The topic of dynamo mode excitation is more closely connected with the problems indicated under the heading (2) above. Kinematic $\alpha \Omega$ dynamos are strictly periodic in time. The magnetic field behaves as the Phoenix, arising from the ashes of the previous cycle, continually rehearsing the very same act as before. Mode excitation in such dynamos is trivial. There is one eigenmode with an infinite phase memory. Many authors have remarked that this is unsatisfactory, and one way out is to consider nonlinear theory. Another possibility is to no longer neglect the 'rest terms' in the dynamo equation, which act as random forcing terms. In this way, too, other modes may be excited. This topic is closely related to the question whether $\langle\mathbf{B}\rangle$ is a two-scale average or an ensemble average.

Let me give one example in support of my allegation that the restrictions of mean field theory are not always appreciated, and then move on to mode excitation proper. For locally isotropic turbulence $\mathbf{v}$, the dynamo coefficients $\alpha$ and $\beta$ are

$$
\begin{aligned}
& \alpha=-\frac{1}{3} \epsilon_{i j k} \int_{0}^{\infty} d \tau<v_{i}(t) \nabla_{j} v_{k}(t-\tau)>\simeq-<\mathbf{v} \cdot \nabla \times \mathbf{v}>\tau_{c} / 3 ; \\
& \beta=\frac{1}{3} \int_{0}^{\infty} d \tau<v_{i}(t) v_{i}(t-\tau)>\simeq<v^{2}>\tau_{c} / 3,
\end{aligned}
$$

where $\tau_{c}$ is the correlation time of $\mathbf{v}$. The gradient $\nabla_{j} \beta$ contains two correlation functions of the type $\left\langle a_{i} \nabla_{j} b_{i}\right\rangle$ which vanish, being isotropic tensors of rank 1 . It follows that $\nabla \beta=0$ and that a position-dependent $\beta$ must necessarily be tensorial. A similar observation goes for $\alpha$. The physical reason is that isotropic, inhomogeneous turbulence does not exist. The role of anisotropies in the turbulence has been emphasised by Rädler (1980; 1983); see also Moffatt (1983) and Schüssler (1984). An analysis of what errors ensue if we nevertheless employ a position-dependent scalar $\alpha$ and $\beta$ is to my knowledge not available. A better known difficulty is that the validity of $(1.1),(1.2)$ and the dynamo equation $(2.1)$ below requires $v \tau_{c} / \lambda_{c} \ll 1$ (First Order Smoothing Approximation or FOSA; $\lambda_{c}=$ correlation length of $\mathbf{v}$ ), while in reality $v \tau_{c} / \lambda_{c} \sim 1$. I shall have no opportunity to discuss attempts to go beyond FOSA, and refer to Stix (1987) for a review. It is conceivable that problems such as these have added to the failure of mean field models, in the sense that there may exist as yet totally unknown and unexplored corners in parameter space. 


\section{Mode Excitation in Mean Field Theory}

The transport properties of the mean field $<\mathbf{B}>$ are determined by the dynamo equation. Its basic form is (Moffatt 1978; Krause and Rädler 1980):

$$
\partial_{t}<\mathbf{B}>=\mathbf{D} \cdot<\mathbf{B}>+\mathbf{F} ; \quad \mathbf{D}=\nabla \times\left(\mathbf{v}_{0} \times+\alpha-\beta \nabla \times\right) .
$$

Here, $\mathbf{v}_{0}$ is the mean flow, and $\alpha$ and $\beta$ are related to the statistical properties of the turbulent flow $\mathbf{v}$ superposed on $\mathbf{v}_{0}$ as in (1.1) and (1.2). The meaning of $\langle\mathbf{B}>$ is not clear. In the traditional two-scale approach (Moffatt 1978, 1983; Krause and Rädler 1980) $<$ B $>$ is a spatial or time average over an unspecified scale in between the largest (size or period dynamo) and the smallest one (eddy size or turn-over time). The term $\mathbf{F}$ collects all unwanted terms due to the fact that the averaging operator $\langle\cdot\rangle$ usually does not commute with $\nabla, \partial_{t}$, etc. $\mathbf{F}$ is generally neglected on the ground that it is formally of order $\lambda_{c} / R \ll 1$ ( $R=$ radius of the dynamo), but there are now indications that $F$ can be large, see sections 5.1 and 6.

Separating the time, $\langle\mathbf{B}>=\mathbf{b} \exp (\lambda t)$, we find from (2.1):

$$
\mathbf{D} \cdot \mathbf{b}=\lambda \mathbf{b} \text {. }
$$

Eq. (2.2) has a discrete spectrum of eigenvalues $\lambda_{n}$ and eigenfunctions or (eigen)modes $\mathbf{b}_{n}$, determined by the geometry of the system and the boundary conditions via the machinery of classical mathematical physics. The solution of eq. (2.1) is

$$
<\mathrm{B}>=\sum_{n} c_{n} \mathbf{b}_{n}(\mathbf{r}) \exp \left(\lambda_{n} t\right) .
$$

The constants $c_{n}$ are determined by the initial condition. The mode concept is somewhat ambiguous as one may always choose another basis set $\left\{\mathbf{b}_{n}^{\prime}\right\}$. The eigenvalue problem (2.2) has been solved for a great variety of different models (Roberts 1972; Stix 1978, 1981; Moffatt 1978; Krause and Rädler 1980; Rädler 1986a; Parker 1979, 1987 and references therein). For an infinite, homogeneous dynamo

$$
\begin{aligned}
& \operatorname{Re} \lambda \simeq(\alpha k \Delta \Omega / 2)^{1 / 2}-\beta k^{2}, \\
& \operatorname{Im} \lambda \simeq(\alpha k \Delta \Omega / 2)^{1 / 2} .
\end{aligned}
$$

The most important change for a finite, inhomogeneous dynamo is that the wave vector $k$ is quantised and approximately equal to $2 \pi /$ (wavelength eigenmode). Furthermore, $\alpha$ and $\beta$ are understood to be typical values and $\Delta \Omega=\left|\nabla v_{0}\right| \sim$ difference in angular speed in the convection zone; $\Delta \Omega \gg \alpha k$ is assumed ( $\alpha \Omega$ approximation). According to (2.4) differential rotation and $\alpha$-effect amplify, while turbulent mixing $(\beta)$ damps the mode. $\operatorname{Re} \lambda$ is a decreasing series, see Fig. 1; its relative position with respect to $\operatorname{Re} \lambda=0$ is determined by the dynamo number $D$ :

$$
D=\alpha \Delta \Omega R_{\odot}^{3} / 2 \beta^{2} .
$$

$\operatorname{Re} \lambda=0$ implies $\left(k R_{\odot}\right)^{3} \simeq D$. Modes with smaller (larger) $k$ are unstable (damped); the smallest $k$ is about $2 \pi / R_{\odot}$. The critical value of $D$ is therefore of 


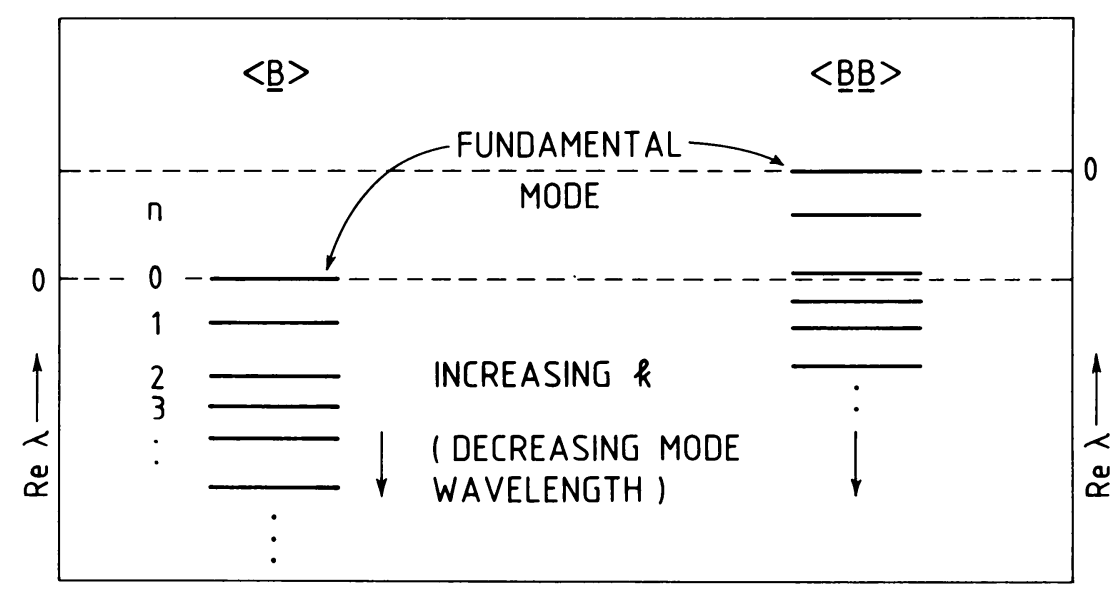

FIG. 1. Growth rates of the eigenmodes $\mathbf{b}_{n}$ of $\langle\mathbf{B}\rangle$. Increasing $n$ means larger $k$ or smaller 'wavelength'. The zero level is determined by $D$. When $D=D_{c}$, the largest growth rate of $\langle\mathbf{B}\rangle$ is zero (marginally stable dynamo). However, $\langle\mathbf{B B}\rangle$ still has a linear instability (section 5.1). Right vertical axis: $\langle$ BB $>$ can be made marginally stable by decreasing $D$ (typically by a factor of order 1) so that $D<D_{c}$. In practice this means that $\beta$ is increased, as $\alpha \Delta \Omega$ is fixed by (2.5). (after Hoyng 1988).

the order of $D_{c} \simeq(2 \pi)^{3}$. For simplicity I ignore the negative branch of $D$. Just as in any system described by a differential equation, also here two mode excitation mechanisms may be distinguished:

(a). Instability $\left(D \geq D_{c}\right)$. One or more modes have $\operatorname{Re} \lambda>0$. These then grow spontaneously from noise, until the validity of (2.1) breaks down. An idea often invoked in the context of mean field theories is that 'nonlinear effects' change the parameters until $D=D_{c}$ (e.g. B may reduce the helicity and hence $\alpha$ ). The fundamental mode is then marginally stable and may be excited at a constant amplitude. The period is $P=2 \pi / \operatorname{Im} \lambda \sim\left(4 \pi R_{\odot} / \alpha \Delta \Omega\right)^{1 / 2}$. Hence, $\alpha \Delta \Omega$ is fixed by requiring $P=22 \mathrm{yr}$, and $\beta$ follows from $\operatorname{Re} \lambda=0$. Overtones have shorter periods and are not excited as they have $\operatorname{Re} \lambda<0$. Such a dynamo would have a single frequency and an infinite quality factor $Q=\omega / \Delta \omega$, as the frequency uncertainty $\Delta \omega$ is zero. However, the fine tuning of $D$ need not be stable. Many authors assume that $D>D_{c}$, so that overtones are excited. Complicated nonlinear interactions between modes and the velocity field may occur which restrict the amplitudes. In that case the dynamo has several frequencies, each with a finite $Q$.

(b). External forcing $\left(D<D_{c}\right)$. In that case all $\operatorname{Re} \lambda<0$ so that according to (2.3), $<\mathbf{B}>\rightarrow 0$. Subcritical mode excitation may occur if $\mathbf{F}$ in (2.1) is sufficiently large. $\mathbf{F}$ depends on $\mathbf{v}$ and thus has in principle the character of a random forcing term. Writing $\mathbf{F}=\Sigma f_{n}(t) \mathbf{b}_{n}$ and $\langle\mathbf{B}\rangle=\Sigma \phi_{k}(t) \mathbf{b}_{k}$, and supposing for simplicity the eigenfunctions $b_{n}$ to be orthonormal, we find from (2.1) and (2.2): 


$$
\dot{\phi}_{k}=\lambda_{k} \phi_{k}+f_{k} \quad \rightarrow \quad<\mathbf{B}>=\sum_{k} \mathbf{b}_{k} \int_{0}^{\infty} d \tau \exp \left(\lambda_{k} \tau\right) f_{k}(t-\tau) .
$$

Hence those modes for which $f_{k} \neq 0$ (possibly all modes) are excited and each would have a finite $Q$ (finite phase memory). Note that $D<D_{c}$ provides merely a lower limit for $\beta$; the numerical value of $\beta$ is no longer fixed. Note further that $D<D_{c}$ does not imply that nonlinear interactions are unimportant.

Another external forcing mechanism would be a relic field in the radiative core, which imposes a nonzero boundary condition on eq. (2.1) at the base of the convection zone. This boundary condition could be periodic, in which case the phase stability of the dynamo at that frequency may be very high.

\section{Observations}

Many solar parameters are now known to vary with the magnetic cycle: luminosity (Hudson 1988; Willson and Hudson 1988), the differential rotation (torsional oscillations; Howard 1984), modulations in the meridional circulation (Ribes and Laclare 1988), the size distribution of surface magnetic flux (Zwaan 1987), and maybe the frequencies of solar oscillations (Gough 1988a; Gelly et al. 1988). I shall restrict myself below to observations which pertain directly to dynamo mode excitation.

\subsection{VARIATIONS IN THE 22-YEAR PERIOD}

The 22 yr magnetic cycle shows fairly large variations in the period lengths. From the epochs of sunspot extrema (Allen 1973) one finds $\delta P_{r m s} / P \sim 0.1$, where $P=$ mean half cycle period. Are subsequent period variations $\delta P$ independent, or is the cycle rather a passive and noisy reflection of a high $Q$ oscillator in the solar core? In the former case there would be a progressive loss of phase memory; in the latter case the phase is locked and never far away from the phase of the core oscillation. Little progress has been made on this old question. Yule (1927) suggested that the phase memory is finite. Dicke (1978) analysed the epochs of sunspot number extrema and concluded that the data indicate phase locking. In a recent study, Dicke (1988) upheld his position. He also found that the transit time for magnetic flux through the convection zone is $\sim 12 \mathrm{yr}$, which is indeed of the order of the turbulent diffusion time $d^{2} / \beta$ over the depth $d$ of the convection zone. However, Gough $(1978,1981$, 1987) made a very similar analysis of the same data and concluded he could not decide either way. If anything, he found a preference for a random walk in phase. Whitehouse (1985) argues that both models are too simple, as he finds evidence for systematic variations in the cycle period.

Barnes et al. (1980) have simulated yearly sunspot numbers from narrowband Gaussian noise and obtained a remarkable similarity with the true cycle. On a longer timescale they also see protracted sunspot minima as during the Maunder Minimum (Eddy 1976, 1983). It appears that the data set (AD 1610 - present) is not sufficient to draw a conclusion regarding the phase stability. Unfortunately (for solar physics), the Precambrian Elatina sediment data are no longer believed to contain an extended solar cycle chronology (Williams 1981, 1985; Sonett and 
Williams 1987), but are now attributed to lunar-induced variations in tidal deposits (Williams 1989; Sonett et al. 1988). The phase stability of the $22 \mathrm{yr}$ cycle may ultimately be determined by a careful analysis of the ${ }^{14} \mathrm{C}$ data and of the ${ }^{10} \mathrm{Be}$ record discovered in Greenlandic ice cores (Beer et al. 1988).

Advanced techniques exist today to determine whether an irregular time series is stochastic or contains a chaotic attractor of low dimension (Schuster 1988; Atmanspacher et al. 1988). For example, Voges et al. (1987) have analysed the $\mathrm{X}$-ray variability of $\mathrm{Her} X-1$ and found an attractor of dimension $2<D_{2}<3$ in the lightcurve. This suggests that the underlying accretion process may be modeled with only 3 nonlinearly coupled variables (it does not tell which, though). Attractor dimensions have been determined from the sunspot and ${ }^{14} \mathrm{C}$ records (Gissatullina et al. 1989; Ostryakov and Usoskin 1989) but these are not yet very reliable. The problem is again the length and quality of the data set (Smith 1988).

\subsection{OTHER PERIODICITIES}

The sunspot data suggest a $\sim 90$ year amplitude modulation (Cohen and Lintz 1974). Tree-ring ${ }^{14} \mathrm{C}$ data and ${ }^{10} \mathrm{Be}$ records indicate irregular modulations in the level of solar activity with a typical timescale of a few hundred years (Sonett 1983a; Stuiver and Braziunas 1988; Raisbeck and Yiou 1988). Interesting new results have been reported on short periods. Stenflo and Vogel (1986) and Stenflo and Weisenhorn (1987) have decomposed the radial surface field $B_{r}$ in spherical harmonics using 25 years of synoptic daily magnetograph data of Mt. Wilson and Kitt Peak. The advantage of this technique is that the results permit a direct comparison with theoretical predictions. The power spectra of the axisymmetric spherical harmonic coefficients $(m=0)$, Fig. 2, show a decoupling between even and odd $l$. The 22 yr dynamo wave is apparently a linear combination of odd- $l$ components. The absence of higher harmonics indicates that the (surface effect of the) wave is almost sinusoidal in time. Decoupling between even and odd $l$ occurs when $\alpha$ is an odd function of colatitude $\theta$; often $\alpha \propto \cos \theta$ is assumed (Moffatt 1978). The data thus confirm this to be basically correct. Further confirmation might come from comparing the observed amplitudes and phases with theoretical odd- $l$ expansions of the fundamental mode. An unexplained feature is that the power distribution becomes abruptly irregular above $l=14$. Stenflo and Vogel (1986) suggest that the power at even $l$ in Fig. 2 indicates a resonant modal structure reminiscent of solar p-mode ridges. Hoyng $(1987 \mathrm{~b}, 1988)$ has interpreted this in terms of excitation of dynamo wave overtones. Taking $k \sim l / R_{\odot}$ one finds with (2.5):

$$
\omega \sim\left(\alpha \Delta \Omega / 2 R_{\odot}\right)^{1 / 2} l^{1 / 2}
$$

which describes the power ridge in Fig. 2 fairly well for $\alpha \Delta \Omega \sim 1.7 \times 10^{-4} \mathrm{~cm} \mathrm{~s}^{-2}$. This is a rather large value and one might therefore speculate that the power at even $l$ reflects dynamo waves of a diffuse dynamo in the convection zone (where $\alpha$ is large), as opposed to dynamo waves of a boundary layer dynamo which we would then see at odd $l$ and in the butterfly diagram. Such an idea had been advanced 


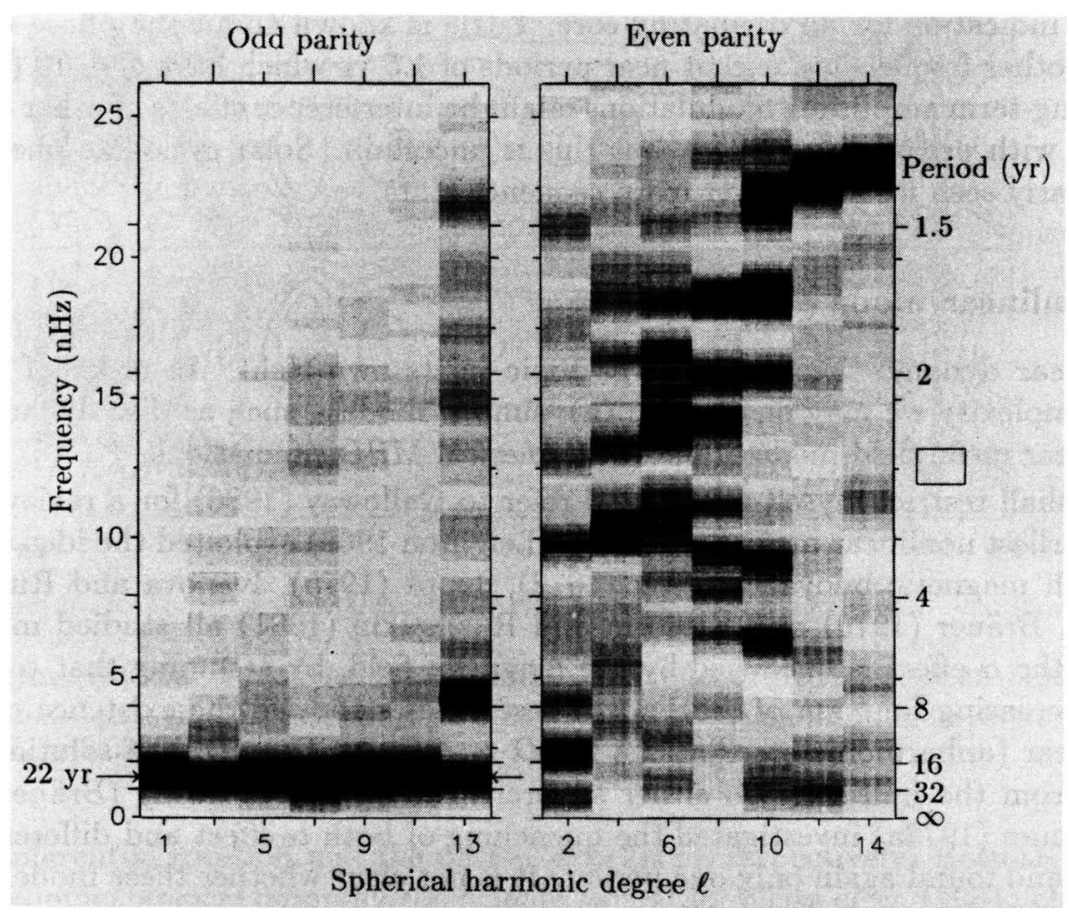

FIG. 2. Power spectrum of the axisymmetric component of the solar radial surface field. Each column is independenty normalised; the average power in even $l$ is about 5 times larger than in odd $l$. (from Stenflo and Weisenhorn 1987).

earlier by Spruit et al. (1987); see also Golub et al. (1981) and Durney (1988). Stenflo and Güdel (1988) report that there is in fact weak power at odd $l$ which interpolates fairly well with the power at even $l$ in Fig. 2, albeit at a systematically lower frequency. Recently, Gokhale and Javaraiah (1989a,b) have confirmed the conclusions of Stenflo and coworkers using magnetic cycle data simulated from the Greenwich sunspot data, which cover a much longer period.

The idea that the magnetic sector structure and coronal holes (Zirker 1977; Hundhausen 1977) may correspond to nonaxisymmetric dynamo modes is relatively old (Stix 1971). These modes, being overtones, are difficult to excite in the linear theory (Stix 1971; Rädler 1986b; Ruzmaikin et al. 1988), but nonlinearities or external forcing can in principle do the job. Other lower main sequence stars are now believed to possess cycles analogous to the solar $11 \mathrm{yr}$ cycle (Vaughan 1983; Baliunas and Vaughan 1985), with periods ranging from $2.6 \mathrm{yr}$ to $\gtrsim 20 \mathrm{yr}$. Very active stars may have more than one period or behave erratically.

In summary, we have several indications that the solar dynamo is multiperiodic. In principle, both nonlinear effects and stochastic excitation may account for that. The phase stability of the $22 \mathrm{yr}$ period is not well known. Its quality factor may be 
as small as $Q=P / \delta P_{r m s} \sim 10$, but could also be much higher. That would be a strong indication for an oscillating core. Little is known about the phase stability at the other frequencies, except near periods of $1.5 \mathrm{yr}$ which have $Q \sim 10$ (Fig. 2). The long-term amplitude modulations might be interference effects of other dynamo modes with periods near $22 \mathrm{yr}$, but this is uncertain. Solar cycle-like phenomena are clearly seen in other lower main sequence stars.

\section{Nonlinear mode excitation}

Nonlinear dynamo theory is a large topic in its own right. In order of increasing complexity we may distinguish (a) 'simple' models, such as disc dynamos, (b) nonlinear mean field models, and (c) numerical MHD simulations.

I shall restrict myself to (b), and refer to Galloway (1986) for a review of (c). The earliest nonlinear mean field model (Leighton 1969) exploited the idea of losses through magnetic buoyancy. Stix (1972), Jepps (1975), Ivanova and Ruzmaikin (1977), Bräuer (1979) and Kleeorin and Ruzmaikin (1981) all studied models in which the $\alpha$-effect is quenched by the magnetic field, by assuming that $\alpha$ in (1.1) is a decreasing function of $|<B>|$. These studies showed the existence of stable nonlinear (anharmonic) oscillations for $D>D_{c}$. At $D=D_{c}$ the solution bifurcates from the null solution either supercritically or subcritically (Bräuer 1980). Yoshimura (1978a) investigated the quenching of both $\alpha$-effect and differential rotation and found again only one period. It is not clear whether these models admit indeed only one period since the exploration of their parameter spaces may have been rather incomplete. Yoshimura (1978b) obtained the first multimodal mean field model by introducing a time delay in the nonlinear coupling. A single delay time produced a regular modulation of the $22 \mathrm{yr}$ cycle, and with two or more different delay times a more chaotic long-term modulation resulted. The reason for the delay is that the Lorentz force produces an acceleration and it will take some time before $\mathbf{v}$ and $\mathbf{v}_{0}$, on which all field regeneration depends, have changed. A time delay therefore effectively increases the order of the equations (= number of independent variables). The early nonlinear work has been reviewed by Stix (1981).

Mean field theory got itself entangled in the net of nonlinear dynamics after Zeldovich and Ruzmaikin had shown how an axisymmetric $\alpha \Omega$ dynamo with nonlinear $\alpha$-effect quenching can be crudely modeled by the Lorenz equations (Ruzmaikin 1981; Jones 1983; see also Krause and Roberts 1981). These equations are wellknown for possessing chaotic solutions (Martens 1984; Schuster 1988). The solution is quasiperiodic but occasionally the trajectory in phase space lingers for a long time near the origin. Accordingly, it was suggested that the Maunder minimum and other 'grand minima' might correspond to a strange attractor (Ruzmaikin 1981), as Yoshimura (1978b) had done implicitly before. This idea was elaborated by Weiss et al. (1984) who included buoyant losses and quenching of differential rotation in addition to $\alpha$-effect suppression. They derived a 7 th order system of equations with two field variables and two variables $\omega_{0}$ and $\omega$ representing the constant part 


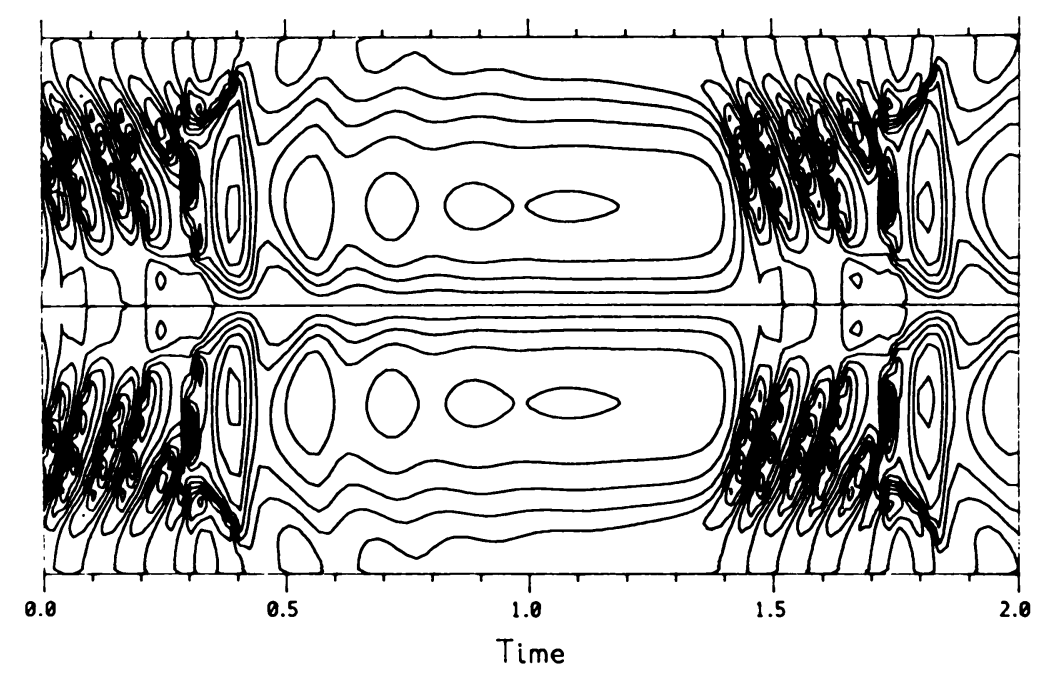

FIG. 3. Butterfly diagram of the nonlinear dynamo model of Belvedere et al. (1989). On the horizontal axis time in units of $R^{2} / \beta$ (154 year). The magnetic Prandtl number is 0.1 and $D / D_{c}=21.3$.

of the differential rotation and the part $\propto \exp (2 i k x)$, respectively. Both $\omega_{0}$ and $\omega$ have phenomenological damping coefficients $\nu_{0}$ and $\nu$. Weiss et al. (1984) obtained their most interesting results for the 6th order system with $\nu_{0}=\infty\left(\omega_{0}=0\right)$, and buoyant losses and $\alpha$-effect quenching switched off. They then observe a series of bifurcations as $D$ increases from $D_{c}$, and at each a new frequency appears in the solution. Beyond $D / D_{c}=3.84$ the solution is chaotic (for $\nu / \beta k^{2}=0.5$ ), featuring irregularly modulated cycles, and episodes with almost zero field. For $D / D_{c}=4$ the interval between such 'grand minima' is about 11.5 average cycle periods.

Model equations such as those of Ruzmaikin (1981) and Weiss et al. (1984) can merely give some indication of the behaviour of real dynamos, since the spatial structure has been severely truncated. As Weiss et al. realised, they have only marginally succeeded in this regard. Chaos and 'grand minima' appear only for $\nu \ll \nu_{0}$ while physically $\nu>\nu_{0}$ is expected. Moreover, the more complete 7 th order system exhibits just aperiodic oscillations without protracted minima. In hindsight it is tempting to regard such studies as a concession to fashion. In those days every respectable field of research had to have its own strange attractor. However, it took almost 5 years before more complete studies were carried out.

Deluca and Gilman $(1986,1988)$ formulated the first mean field boundary layer dynamo, featuring a selfconsistent mean flow $\mathbf{v}_{0}$, but phenomenological $\alpha$-effect quenching and flux losses. They found steady and time-varying solutions (depending very sensitively on the parameter values), but no periodic dynamo waves. They concluded that the magnetic field is unable to generate sufficient shear to drive an 
$\alpha \Omega$ dynamo. Schmitt and Schüssler (1989) studied a mean field boundary layer dynamo with either $\alpha$-effect suppression or flux loss, and concluded that the latter is likely to be the more important of the two. They noted that the mode in which a dynamo finds itself may well depend on its history, since there are sometimes several possible modes for a given $D$. This is also reported by Brandenburg et al. (1989) and Belvedere et al. (1989), who argue that this implies that there could be large differences in the dynamo parameters (period, activity level) of otherwise very similar stars. This is indeed observed in late type stars. However, it is not clear if these coexisting solutions are all stable. This point has been investigated by Jennings and Weiss (1989).

Brandenburg et al. (1989) analysed a mean field $\alpha \Omega$ dynamo in the convection zone, incorporating only $\alpha$-effect quenching, $\alpha=\alpha_{0} \cos \theta /\left\{1+\left\langle\mathbf{B}>^{2}\right\}\right.$. They found periodic single mode solutions, but unlike the earlier works of Jepps (1975) and Yoshimura (1978a) which contain much the same physics, an overall amplitude modulation was observed in certain narrow intervals of $\alpha_{0}$. The field structure is then largely dipolar, but changes into a quadrupole when the amplitude is minimal. Belvedere et al. (1989) extended the work of Weiss et al. (1984) and investigated a mean field dynamo in which the only nonlinearity is a selfconsistent variation in the mean flow on top of a given differential rotation. The equations are truncated in $r$, but full $\theta$ dependence is retained. The ensuing gain in computing time enabled a systematic investigation of the transition between the various types of solution. For $D / D_{c} \lesssim 4.3$ there is a singly periodic solution, for $D / D_{c} \gtrsim 4.3$ multiperiodic solutions appear on stage, while for $D / D_{c} \gtrsim 8.5$ the solution features 'relatively long periods of stasis, interrupted by interludes of cyclical behaviour', see Fig. 3.

It is clear that nonlinear mean field theory is still in a very early stage of development. The various studies do not yet show a clear pattern of commonality. It is not known which of the nonlinearities are the more important ones, and the solutions are very sensitive to the functional form of the nonlinear coupling and/or the values of parameters. The main defect is that the nonlinearities are introduced phenomenologically, since dynamically consistent expressions for their dependence on $\langle\mathbf{B}>$ are not available. Investigations to derive these functional forms from first principles are presently of the utmost importance (cf. Malkus and Proctor 1975; Moffatt 1978; Zeldovich et al. 1983). The following illustrates some of the problems lying ahead. All authors considering a selfconsistent mean flow $\mathbf{v}_{\mathbf{0}}$ write the mean Lorentz force as $\left\langle f_{i}\right\rangle=\{(\nabla \times<\mathrm{B}>) \times<\mathbf{B}>\}_{i} / 4 \pi$, while actually

$$
<f_{i}>=<(\nabla \times \mathrm{B}) \times \mathrm{B}>_{i} / 4 \pi=\nabla_{j}\left(2 T_{i j}-T_{k k} \delta_{i j}\right) / 8 \pi .
$$

Hence, $\left\langle f_{i}\right\rangle$ depends on a higher average $T_{i j}=\left\langle B_{i} B_{j}\right\rangle$. Both expressions coincide if the fluctuating field $\mathbf{B}^{\prime}=\mathbf{B}-\langle\mathbf{B}>$ is small, but that is not the case in the Sun. Only when hurdles such as these have been taken, may we begin to investigate in detail the relations between truncated mode equations and the full nonlinear partial differential equations, as has been done for example by Moore et al. (1983) and Knobloch et al. (1986) in their study of two-dimensional thermosolutal convection. 


\section{Mode excitation by external forcing}

External forcing comes in two varieties: through boundary conditions or fluctuations in the turbulent convection. I shall briefly discuss the former and then deal with the second topic. It has long been speculated that the radiative core of the Sun may contain a relic magnetic field. If such a field exists, it imposes a boundary condition $\mathbf{B}=\mathbf{B}_{\text {core }}$ at the base of the convection zone. The field will be smeared out by the turbulent dynamo, but there will be a net polarity and intensity asymmetry in the activity cycle: alternate halves of the full $22 \mathrm{yr}$ cycle have different amplitudes. Sonett (1983b) analysed the sunspot record from this point of view and found $\mathrm{a} \sim 4 \%$ intensity asymmetry. If caused by a fossil dipole field, its strength was estimated to be less than 0.6 G. Levy and Boyer (1982) and Boyer and Levy (1984) analysed kinematic mean field models with a dipolar or quadrupolar field imposed at the base of the convection zone. The dynamo reduces the externally visible magnetic moment by about a factor 3 . They also concluded that the poloidal component of the relic field can be no more than a few gauss. Pudovkin and Benevolenska (1985) attribute the occurrence of 'grand minima' to a periodic $\mathbf{B}_{\text {core }}$ at the bottom of the convection zone $(P=180 \mathrm{yr})$.

Piddington $(1971,1976)$ and Layzer et al. (1979) have sketched oscillator theories for the solar cycle. Piddington visualizes a $22 \mathrm{yr}$ torsional oscillation of the entire core with the poloidal component of the field acting as a spring, while Layzer et al. suggest that the oscillation is restricted to the transition layer between core and convection zone. A $22 \mathrm{yr}$ period requires fields of $\sim 100 \mathrm{G}$. Unfortunately, an acceptable model has never been presented (for a detailed critique I refer to Cowling 1981), and it could be worthwile to try and see if this can be done. It is after all not excluded that the entire Sun performs a $22 \mathrm{yr}$ oscillation of which the magnetic cycle is only one manifestation (Gough 1988b). The dynamo in the convection zone could then be locked in to this oscillation (e.g. by a boundary condition) and produce a somewhat noisy surface effect which has the same $Q$ as the driver.

\subsection{STOCHASTIC EXCITATION}

External forcing by fluctuations in the turbulence has received minimal attention. Parker (1969) and Levy (1972) suggest that a sudden burst in the cyclonic convection causes a jump in $\alpha$, and showed that this may induce a reversal of the (mean) geomagnetic field. This explanation is essentially ad hoc, and the conventional wisdom of today is that such things as reversals in $\alpha^{2}$ dynamos and aperiodicity in $\alpha \Omega$ dynamos are caused by nonlinearities. However, even in linear theory the periods of adjacent cycles of an $\alpha \Omega$ dynamo must differ, as the realisation of the turbulence is different. It should be possible to formulate linear mean field theory in the same way as a scalar diffusion process, with a continuous loss of memory.

This problem has been taken up by Hoyng $(1987 a, b)$. To be able to evaluate the effect of the fluctuations hidden in $F$ in eq. (2.1) he interpretes $\langle\cdot\rangle$ as an ensemble average. In that case $\mathbf{F}=0$, since $\partial_{t}, \nabla$, etc., commute exactly with $<\cdot>$. $\dagger$ The ensemble average is best understood literally, as an average over many 
copy systems, e.g. $\left\langle q>_{\mathbf{r}, t}=\lim _{N}\left\{\sum_{i} q_{i}(\mathbf{r}, t)\right\} / N\right.$, where $q$ is an arbitrary physical quantity, cf. Krause (1976). Fluctuations now cause phase mixing: $\mathbf{B}$ evolves independently in each system and, in the case of the Sun, the 'copy suns' will distribute themselves evenly over the magnetic cycle (after some time), whence $<$ B $>\rightarrow 0$. Hoyng concludes that (1) only a damped solution of (2.1) is physically meaningful, and (2) the ensemble average $\langle\mathrm{B}\rangle$ has no relation with observable fields; in particular, it is not equal to the large-scale field. Accepting a damped solution for $<\mathrm{B}>$ implies decreasing $D$ so that $D<D_{c}$. The freedom in $\beta$ is used to remove the linear instability of $\langle$ BB $\rangle$ (Parker 1979, §17.6), see Fig. 1. The damping time of mode $b_{n}$ is interpreted as a measure of its phase stability. For the dipole mode of a simple $\alpha^{2}$ dynamo with constant $\alpha$ and $\beta$ this time is only $\sim 0.15 R^{2} / \beta$, less than the timescale for turbulent diffusion through the sphere! It is argued that the dipole component of $\mathbf{B}$ must rapidly wander over the sphere, contrary to the traditional view which suggests that this $\alpha^{2}$ dynamo possesses a constant field (Krause and Rädler 1980, Ch. 14).

Since $<\mathbf{B}>$ is no longer indicative for the field $\mathbf{B}$ of the dynamo, Hoyng (1988) proposes to expand $B$ in terms of the eigenfunctions $\mathbf{b}_{n}$ :

$$
\mathbf{B}=\sum_{n} c_{n}(t) \mathbf{b}_{n}(\mathbf{r})
$$

The coefficients $c_{n}(t)$ determine the evolution of large scale fields (small $n$ ) as well as small scale fields ( $n$ large). For an $\alpha \Omega$ dynamo like the Sun, each $c_{n}(t)$ turns out to be a quasi-periodic random function whose mean period and coherence time are roughly given by the eigenvalue $\lambda_{n}$ of $b_{n}$. The power spectrum of $c_{n}(t)$ is:

with

$$
P_{n}(\omega) \sim \frac{\delta_{n}}{\pi}<\left|c_{n}\right|^{2}>\left[\frac{1}{\left(\omega-\omega_{n}^{\prime}\right)^{2}+\delta_{n}^{2}}+\frac{1}{\left(\omega+\omega_{n}^{\prime}\right)^{2}+\delta_{n}^{2}}\right]
$$

$$
\omega_{n}^{\prime} \sim \omega_{n} \equiv \operatorname{Im} \lambda_{n} ; \quad \delta_{n} \sim-\operatorname{Re} \lambda_{n}
$$

Hence, all eigenmodes turn out to be excited, with a finite frequency stability $Q_{n}=\omega_{n}^{\prime} / \Delta \omega_{n}^{\prime} \sim \omega_{n} / \delta_{n}$, cf. section 2 and Fig. 4 . The frequencies $\omega_{n}^{\prime}$ are shifted from their unperturbed position $\omega_{n}$ because the modes are driven. At present only an estimate of the frequency stability of the $22 \mathrm{yr}$ cycle is available, $Q \sim 1$ (Hoyng $1987 \mathrm{~b}$ ), which is so small that there would be hardly a periodic cycle left.

The theory is currently being applied to the solar dynamo and it seems too early to judge its merits before that has been done. A salient feature is the potentially very drastic influence of the fluctuations: If this turns out to be true then one may have to look for nonlinear effects (yet to be included) as a stabilising factor, the reverse of their traditional role. Another feature is that the eigenfunctions $\mathbf{b}_{n}$ have lost their pretension of representing the dynamo field; they are just a (very handy)

$\dagger$ This is also true if $\langle\cdot\rangle$ is a longitudinal average (Braginskii 1965a,b). I conjecture that in this case the neglect of fluctuations appears in the same way as it does for ensemble averages: a nonzero and finite $\langle\mathbf{B}\rangle$ implies $\langle\mathbf{B B}\rangle \rightarrow \infty$ (Hoyng 1987b). 

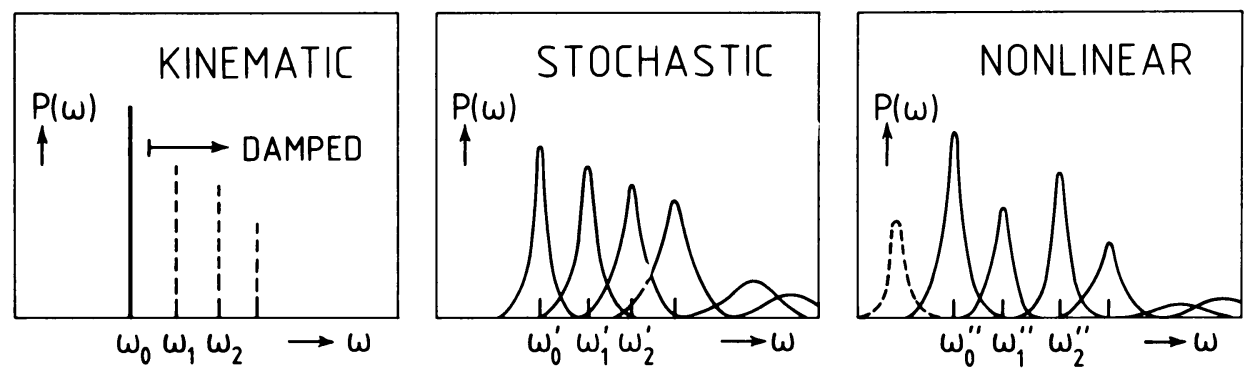

FIG. 4. Left: a marginally stable linear $\alpha \Omega$ dynamo has $D=D_{c}$ and possesses one frequency and $Q=\infty$. Middle: according to the linear stochastic theory $D<D_{c}$ and all eigenmodes are excited, each with a finite $Q$; the frequencies $\omega_{n}^{\prime}$ are shifted from their unperturbed positions $\omega_{n}$. Right: inclusion of nonlinear effects further changes the line shapes and positions, and very low frequencies may also appear.

set of basis functions. It remains necessary to identify ensemble averages such as $\left\langle c_{n}(t)\right\rangle,\left\langle\left|c_{n}(t)\right|^{2}\right\rangle$, with time averages $\overline{c_{n}(t)}, \overline{\left|c_{n}(t)\right|^{2}}$, but since only scalars are involved depending on time, this is far less controversial than for $\mathbf{B}$.

\section{Discussion}

In Fig. 4, I have summarized my view on the evolution of mean field dynamo theory. In the early days, the notion of marginal stability $\left(D=D_{c}\right)$ was implicitly accepted, for lack of better. Next came nonlinear dynamos operating at $D>D_{c}$. These feature in principle many nonlinearly interacting magnetic and fluid modes. It has been speculated that their large-scale dynamics can be described adequately with very few modes only and under those circumstances a low dimensional strange attractor might emerge. But if and when this occurs is unknown, also from the point of view of observations. Theoretically, the main obstacle is the lack of selfconsistent nonlinearities. I have pointed out an additional problem, that $<\mathbf{B B}>$ diverges due to the neglect of fluctuations. In the stochastic excitation picture the dynamo would operate subcritically $\left(D<D_{c}\right)$, and $<\mathrm{BB}>$ no longer diverges. Many modes are excited and their evolution is coupled. The dimension of the relevant part of phase space (and of attractors) is very high. The situation is analogous to the classical (linear) theory of Brownian motion, and the theory may indeed be regarded as a linear theory for the random walk of an advected vector field. Of course, this idea must still be tested in real dynamo models. Also nonlinear effects remain to be included, and here we face again the problem of selfconsistency.

In moments of complacency one might be inclined to think that dynamo theory has come a long way since the idea of a hydromagnetic dynamo first arose (Larmor 1919) and the pioneering contributions of Cowling (1934), Parker (1955) and Steenbeck, Krause and Rädler (Roberts and Stix 1971). But in fact I don't think it has. I believe we still have a much longer way to go. 


\section{Acknowledgements}

I am indebted to many colleagues for their help, critical comments and for sending me their recent work: A.A. van Ballegooijen, G. Belvedere, A. Brandenburg, F.H. Busse, S. Childress, A. Gailitis, J.H.G.M. van Geffen, P.A. Gilman, E. Knobloch, M. Meneguzzi, M.R.E. Proctor, K.-H. Rädler, E. Ribes, P.H. Roberts, M. Schüssler, A.M. Soward, J.O. Stenflo, M. Stix and N.O. Weiss.

\section{References}

Allen, C.W.: 1973, Astrophysical Quantities, Athlone Press (London).

Atmanspacher, H., Scheingraber, H. and Voges, W.: 1988, Phys. Rev. A37, 1314.

Baliunas, S.L. and Vaughan, A.H.: 1985, Ann. Rev. Astron. Astrophys. 23, 379.

Barnes, J.A., Sargent, H.H. and Tryon, P.V.: 1980, in The Ancient Sun, eds. R.O. Pepin, J.A. Eddy and R.B. Merrill, Pergamon Press (New York), p. 159.

Beer, J., Siegenthaler, U. and Blinov, A.: 1988, in Secular Solar and Geomagnetic Variations in the Last 10,000 Years, eds. F.R. Stephenson and A.W. Wolfendale, Kluwer Academic Publishers (Dordrecht), p. 297.

Belvedere, G., Pidatella, R.M. and Proctor, R.M.E.: 1989, Geophys. Astrophys. Fluid Dynamics, to appear.

Boyer, D.W. and Levy, E.H.: 1984, Astrophys. J. 277, 848.

Braginskii, S.I.: 1965, Sov. Phys. JETP 20, 726.

Braginskii, S.I.: 1965, Sov. Phys. JETP 20, 1462.

Brandenburg, A., Moss, D. and Tuominen, I.: 1989, Geophys. Astrophys. Fluid Dynamics, to appear.

Bräuer, H.: 1979, Astron. Nachr. 300, 43.

Bräuer, H.: 1980, Astron. Nachr. 301, 203.

Cohen, T.J. and Lintz, P.R.: 1974. Nature 250, 398.

Cowling, T.G.: 1934, M.N.R.A.S. 94, 39.

Cowling, T.G.: 1981, Ann. Rev. Astron. Astrophys. 19, 115.

Deluca, E.E. and Gilman, P.A.: 1986, Geophys. Astrophys. fluid Dynamics 37, 85.

Deluca, E.E. and Gilman, P.A.: 1988, Geophys. Astrophys. fluid Dynamics 43, 119.

Dicke, R.H.: 1978, Nature 276, 676.

Dicke, R.H.: 1988, Solar Phys. 115, 171.

Durney, B.R.: 1988, Astron. Astrophys. 191, 374.

Dziembowski, W.A., Goode, P.R. and Libbrecht, K.G.: 1989, Astrophys. J. 337, L53.

Eddy, J.A.: 1976, Science 192, 1189.

Eddy, J.A.: 1983, Solar Phys. 89, 195.

Galloway, D.J.: 1986, Adv. Space Res. 6, No. 8, 19.

Galloway, D.J. and Weiss, N.O.: 1981, Astrophys. J. 243, 945.

Gelly, B., Fossat, E. and Grec, G.: 1988, in Seismology of the Sun \& Sun-like Stars, ed. E.J. Rolfe, ESA SP-286 (Noordwijk), p. 275.

Gilman, P.A.: 1983, Astrophys. J. Suppl. 53, 243.

Gilman, P.A. and Miller, J.: 1981, Astrophys. J. Suppl. 46, 211.

Gilman, P.A., Morrow, C.A. and Deluca, E.E.: 1989, Astrophys. J. 338, 528.

Gissatullina, S.M., Rukavishnikov, V.D., Ruzmaikin, A.A. and Tavastsherna, K.S.: 1989, Solar Phys., submitted.

Glatzmaier, G.A.: 1985a, Astrophys. J. 291, 300.

Glatzmaier, G.A.: 1985b, Geophys. Astrophys. Fluid Dynamics 31, 137.

Golub, L., Rosner, R., Vaiana, G.S. and Weiss, N.O.: 1981, Astrophys. J. 243, 309.

Gokhale, M.H. and Javaraiah, J.: 1989a, M.N.R.A.S., submitted.

Gokhale, M.H. and Javaraiah, J.: 1989b, these proceedings. 
Gough, D.O.: 1978, in Pleins Feux sur la Physique Solaire, eds. S. Dumont and J. Rösch, Editions du CNRS (Paris), p. 81.

Gough, D.O.: 1981, in Variations in the Solar Constant, ed. S. Sofia, US Govt. Printing Office (Washington), p. 185.

Gough, D.O.: 1987, in Solar-Terrestrial Relationships and the Earth Environment in the Last Millenia, ed. G. Castalogni-Cini, Soc. Italiana di Fisica (Bologna), p. 95.

Gough, D.O.: 1988a, in Seismology of the Sun \& Sun-like Stars, ed. E.J. Rolfe, ESA SP286 (Noordwijk), p. 679.

Gough, D.O.: 1988b, Nature 336, 618.

Harvey, J.: 1988, in Seismology of the Sun \& Sun-like Stars, ed. E.J. Rolfe, ESA SP-286 (Noordwijk), p. 55.

Howard, R.: 1884, Ann. Rev. Astron. Astrophys. 22, 131.

Hoyng, P.: 1987a, Astron. Astrophys. 171, 348.

Hoyng, P.: 1987b, Astron. Astrophys. 171, 357.

Hoyng, P.: 1988, Astrophys. J. 332, 857.

Hudson, H.S.: 1988, Ann. Rev. Astron. Astrophys. 26, 437.

Hundhausen, A.J.: 1977, in Coronal Holes and High Speed Wind Streams, ed. J.B. Zirker, Colorado Associated U.P. (Boulder), p. 225.

Ivanova, T.S. and Ruzmaikin, A.A.: 1977, Sov. Astron. 21, 479.

Jennings, R.L. and Weiss, N.O.: 1989, these proceedings.

Jepps, S.A.: 1975, J. Fluid Mech. 67, 625.

Jones, C.A.: 1983, in Stellar and Planetary Magnetism, ed. A.M. Soward, Gordon and Breach (New York), p. 159.

Kleeorin, N.I. and Ruzmaikin, A.A.: 1981, Geophys. Astrophys. Fluid Dynamics 17, 281.

Knobloch, E., Moore, D.R., Toomre, J. and Weiss, N.O.: 1986, J. Fluid Mech. 166, 409.

Krause, F.: 1976, in Basic Mechanisms of Solar Activity, eds. V. Bumba and J. Kleczec, Reidel (Dordrecht), p. 305.

Krause, F. and Rädler, K.-H.: 1980, Mean-Field Magnetohydrodynamics and Dynamo Theory, Pergamon Press (London).

Krause, F. and Roberts, P.H.: 1981, Adv. Space Res. 1, 231.

Larmor, J.: 1919, Rep. Brit. Assoc. Adv. Sci. 1919, 159.

Layzer, D., Rosner, R. and Doyle, H.T.: 1979, Astrophys. J. 229, 1126.

Levy, E.H.: 1972, Astrophys. J. 171, 635.

Levy, E.H. and Boyer, D.: 1982, Astrophys. J. 254, L19.

Malkus, W.V.R. and Proctor, M.R.E.: 1975, J. Fluid Mech. 67, 417.

Martens, P.C.H.: 1984, Physics Reports 115, 315.

Moffatt, H.K.: 1978, Magnetic Field Generation in Electrically Conducting Fluids, Cambridge U.P. (Cambridge).

Moffatt, H.K.: 1983, Rep. Prog. Phys. 46, 621.

Moore, D.R., Toomre, J., Knobloch, E. and Weiss, N.O.: 1983, Nature 303, 663.

Ostryakov, V.M. and Usoskin, I.G.: 1989, these proceedings.

Parker, E.N.: 1955, Astrophys. J. 122, 293.

Parker, E.N.: 1969, Astrophys. J. 158, 815.

Parker, E.N.: 1979, Cosmical Magnetic Fields, Clarendon Press (Oxford).

Parker, E.N.: 1987, Solar Phys. 110, 11.

Piddington, J.H.: 1971, Proc. Astron. Soc. Australia 2, 7.

Piddington, J.H.: 1976, in Basic Mechanisms of Solar Activity, eds. V. Bumba and J. Kleczek, Reidel (Dordrecht), p. 389.

Pudovkin, M.I. and Benevolenska, E.E.: 1985, Solar Phys. 95, 381.

Rädler, K.-H.: 1980, Astron. Nachr. 301, 101.

Rädler, K.-H.: 1983, in Stellar and Planetary Magnetism, ed. A.M. Soward, Gordon and Breach (New York), p. 17 and p. 37.

Rädler, K.-H.: 1986a, Astron. Nachr. 307, 89.

Rädler, K.-H.: 1986b, in Plasma Astrophysics, ESA SP-251 (Noordwijk), p. 569. 
Raisbeck, G.M. and Yiou, F.: 1988, in Secular Solar and Geomagnetic Variations in the Last 10,000 Years, eds. F.R. Stephenson and A.W. Wolfendale, Kluwer Academic Publishers (Dordrecht), p. 287.

Ribes, E. and Laclare, F.: 1988, Geophys. Astrophys. Fluid Dynamics 41, 171.

Roberts, P.H. and Stix, M.: 1971, NCAR-TN/IA-60, (NCAR, Boulder, Colorado).

Roberts, P.H.: 1972, Phil. Trans. Roy. Soc. Lond. A272, 663.

Ruzmaikin, A.A.: 1981, Comm. Astrophys. 9, 85.

Ruzmaikin, A.A., Sokolov, D.D. and Starchenko, S.V.: 1988, Solar Phys. 115, 5.

Schmitt, D. and Schüssler, M.: 1989, Astron. Astrophys., to appear.

Schüssler, M.: 1984, in The Hydromagnetics of the Sun, ESA SP-220 (Noordwijk), p. 67.

Schuster, H.G.: 1988, Deterministic Chaos, VHC Verlagsgesellschaft (Weinheim).

Smith, L.A.: 1988, Phys. Lett. 133A, 283.

Sonett, C.P.: 1983a, Rev. Geophys. Space Phys. 22, 239.

Sonett, C.P.: 1983b, Nature 306, 670 .

Sonett, C.P. and Williams, G.E.: 1987, Solar Phys. 110, 397.

Sonett, C.P., Finney, S.A. and Williams, C.R.: 1988, Nature 335, 806.

Spiegel, E.A. and Weiss, N.O.: 1980, Nature 287, 616.

Spruit, H.C. and Van Ballegooijen, A.A.: 1982, Astron. Astrophys. 106, 58.

Spruit, H.C., Title, A.M. and Van Ballegooijen, A.A.: 1987, Solar Phys. 110, 115.

Stenflo, J.O. and Vogel, M.: 1986, Nature 319, 285.

Stenflo, J.O. and Weisenhorn, A.L.: 1987, Solar Phys. 108, 205.

Stenflo, J.O. and Güdel, M.: 1988, Astron. Astrophys. 191, 137.

Stix, M.: 1971, Astron. Astrophys. 13, 203.

Stix, M.: 1972, Astron. Astrophys. 20, 9.

Stix, M.: 1978, in Pleins Feux sur la Physique Solaire, eds. S. Dumont and J. Rösch, Editions du CNRS (Paris), p. 37.

Stix, M.: 1981, Solar Phys. 74, 79.

Stix, M.: 1987, in Solar and Stellar Physics, eds. E.H. Schröter and M. Schüssler, Springer Verlag (Berlin), p. 15.

Stuiver, M. and Braziunas, T.F.: 1988, in Secular Solar and Geomagnetic Variations in the Last 10,000 Years, eds. F.R. Stephenson and A.W. Wolfendale, Kluwer Academic Publishers (Dordrecht), p. 245.

Vaughan, A.H.: 1983, in Solar and Stellar Magnetic Fields: Origins and Coronal Effects, ed. J.O. Stenflo, Reidel (Dordrecht), p. 113.

Voges, W., Atmanspacher, H. and Scheingraber, H.: 1987, Astrophys. J. 320, 794.

Weiss, N.O., Cattaneo, F. and Jones, C.A.: 1984, Geophys. Astrophys. Fluid Dynamics $30,305$.

Whitehouse, D.R.: 1985, Astron. Astrophys. 145, 451.

Williams, G.E.: 1981, Nature 291, 624 .

Williams, G.E.: 1985, Aust. J. Phys. 38, 1027.

Williams, G.E.: 1989, J. Geol. Soc. London 146, 97.

Willson, R.C. and Hudson, H.S.: 1988, Nature 332, 810.

Yoshimura, H.: 1978a, Astrophys. J. 220, 692.

Yoshimura, H.: 1978b, Astrophys. J. 226, 706.

Yule, G.U.: 1927, Phil. Trans. Roy. Soc. London A226, 267.

Zeldovich, Ya.B., Ruzmaikin, A.A. and Sokoloff, D.D.: 1983, Magnetic Fields in Astrophysics, Gordon and Breach (New York).

Zirker, J.B.: 1977, in Coronal Holes and High Speed Wind Streams, ed. J.B. Zirker, Colorado Associated U.P. (Boulder), p. 1.

Zwaan, C.: 1987, Ann. Rev. Astron. Astrophys. 25, 83. 